\title{
Investigation of the Distributions and Types of Multidrug-Resistant Acinetobacter baumannii in Different Departments in a General Hospital
}

\author{
Yaner Qian ${ }^{1}$; Xuejun Dong ${ }^{1}$; Zongxin Wang ${ }^{1}$; Guocan Yang ${ }^{1}$; Qi Liu ${ }^{1, *}$ \\ ${ }^{1}$ The Center of Clinical Laboratory, Shaoxing People's Hospital, Shaoxing Hospital of Zhejiang University, Shaoxing, 312000, China \\ ${ }^{*}$ Corresponding author: Qi Liu, The Center of Clinical Laboratory, Shaoxing People’s Hospital, Shaoxing Hospital of Zhejiang University, Shaoxing, 312000, China. Fax: +86- \\ 57588228566, E-mail: qiliu_shaoxing@163.com
}

Received: August 20, 2014; Revised: October 31, 2014; Accepted: November 4, 2014

\begin{abstract}
Background:Acinetobacter baumannii is the most prevalent strain in hospitals and different clinical departments.
Objectives: The current study aimed to investigate the genetic characteristics and resistance mechanisms of A. baumannii isolated from clinical samples in Shaoxing people's hospital affiliated to Zhejiang University, Shaoxing, China.

Patients and Methods: Acinetobacter baumannii strains were isolated from blood, phlegm and skin of the patients hospitalized in different departments as respiratory medicine, plastic surgery and intensive care unit (ICU). Multilocus sequence typing (MLST) was used to characterize the isolates. Kirby-Bauer test was used to evaluate antibiotic resistance of the bacteria. The expression of resistance inducing genes was detected by reverse transcription polymerase chain reaction (RT-PCR). The results were analyzed and compared.

Results: Two bacterial types, ST208, and ST218, were identified in all 140 samples. The ST208 mainly came from ICU and department of respiratory medicine, while ST218 from department of plastic surgery; 70.21\% of ST208 and 84.78\% of ST218 were carbapenem-resistant Acinetobacter baumannii (CRAB) and carbapenem-susceptible Acinetobacter baumannii (CSAB), respectively. Multidrug-resistance genes in CRAB isolated from the hospital mainly included, oxa-23, oxa-5, intl 1 and qace $\Delta 1$-sul 1. Besides, the highest and lowest antibiotic resistance was observed in the strains isolated from blood samples and wounds, respectively.

Conclusions: The distribution of $\mathrm{AB}$ varies in different clinical departments and samples. In the hospital under study, the main types of $\mathrm{AB}$ were ST208 and ST218. The genes which affect the ability of antibiotic-resistance were oxa-23, oxa-51, intl 1 and qace $\Delta 1$-sul 1 .
\end{abstract}

Keywords: Acinetobacter baumannii; Antibiotic Resistance; Multilocus Sequence Typing

\section{Background}

Currently, antibiotic resistance is very common. Some Gram-negative bacteria could express extensive multidrug resistance and significantly increase the rate of nosocomial infections in the hospital (1). Among these bacteria Acinetobacter baumannii is the most prevalent species in different geographical areas (2). Previous studies showed that $A$. baumannii was already an emerging pathogen in the respiratory tract, wounds, blood and urine (3). Since the strains of A. baumannii were usually resistant to most available drugs, except colistin and tigecycline, they would be very sticky when detected on a patient. Therefore, the urgent need to study the epidemiology of resistance, surveillance and control measures for A. baumannii in hospital infections was warned (4).

Acinetobacter baumannii could be found in diverse sources such as food, water and soils. It is also located on the skin of healthy human. Acinetobacter baumannii strains are able to survive for a long time even on a dry surface and some of them could toughly attach to human epithelial cells through fimbriae or side chains (5). These characteristics indicate that $A$. baumannii could easily spread via surface, air or any other possible pathways. Moreover, many stud- ies showed that in a certain section of the hospital there was only one type of A. baumannii $(1,6)$. This is quite interesting that different strains may react differently against antibiotics. The sources of the harmful A. baumannii may be found by analyzing the molecular epidemiology of the strain in time. However, there are not enough studies on this subject. On the other hand, although physicians and surgeons all reported the threat of A. baumannii, most of the raised problems were from internal medicine or ICU. The differences may be caused by the selective pressure from indiscriminate use of broad-spectrum antibiotics in different clinical departments (7). Analysis may lead to a new explanation for the formation and spread of carbapenem-resistant Acinetobacter baumannii (CRAB) or carbapenem-susceptible Acinetobacter baumannii (CSAB) (8). However, by now few researches compared the differences of the distribution in different departments. Thus, this issue is worth studying further.

\section{Objectives}

The current study aimed to analyze the distribution of certain types of A. baumannii in clinical departments. Au-

Copyright (C) 2015, Ahvaz Jundishapur University of Medical Sciences. This is an open-access article distributed under the terms of the Creative Commons Attribution-NonCommercial 4.0 International License (http://creativecommons.org/licenses/by-nc/4.0/) which permits copy and redistribute the material just in noncommercial usages, provided the original work is properly cited. 
Qian Yet al.

thors tried to figure out the roles of the antibiotic-resistant genes and the therapies for the diseases in different departments with spread of CRAB and CSAB.

\section{Patients and Methods}

\subsection{Analysis of Acinetobacter baumannii Species Isolated From Clinical Samples}

Clinical samples were collected from January 2010 to January 2014. Eighty-one patients from three wards as ICU, department of respiration medicine, department of burn and plastic surgery were studied. The current study was approved by the medical ethics committee of Zhejiang University, Shaoxing, China. (Code: 201005392). Both oral and written informed consents were taken from every patient in the study. All patients had received at least one type of antibiotic. Blood, sputum and wound swab samples were collected and studied. One hundred-fiftysix $A B$ isolates were isolated from these samples. Details are shown in Table 1 . All strains were identified by the VITEK32 automatic bacteria identification system (M-D Corporation, US). The bacteria were cultured on MuellerHinton (MH) agar medium (Oxoid, UK) and LB broth medium (Shenggong, China) (2).

\subsection{Multilocus Sequence Typing}

MLST was used to study the genetic characteristics of the strains. In detail, cultured isolations were collected and solved in boiling double distilled water for $10 \mathrm{~min}$ utes and then centrifuged at $12000 \mathrm{rpm}$. The supernatant was collected and used as the template for further MLST reaction. The housekeeping genes of $A B ;$ gltA, gyrB, $g d h B, g p i$, and $r p o D$ were selected as the standard template $(9,10)$. The sequences of the primers were designed according to those of the previous studies $(3,11)$ (Table 2). All primers were designed by Jinsi Rui Corporation, China, and induced by RT-PCR kits (DRR063A, Takara, Japan). Polymerase chain reaction (PCR) was performed as follows: Initial denaturation, $95^{\circ} \mathrm{C}$ for 5 minutes; denaturation, $95^{\circ} \mathrm{C}$ for 1 minute; annealing, $56^{\circ} \mathrm{C}$ for 30 seconds; extension, $72^{\circ} \mathrm{C}$ for 45 seconds. There were 35 cycles and each cycle contained three steps (1). At last, the temperature stayed at $72^{\circ} \mathrm{C}$ for 10 minutes. After the PCR reaction, the products were gathered and purified by PCR purification kit (Axygen, AP-PCR-250, Silicon, US). Then the products were analyzed by MLST. This test was performed by Ruibo Corporation, China. The test results were used to identify the strains collected from clinical specimens by referring to the AB MLST database (7-9).

\begin{tabular}{lcccc}
\hline Table 1. Number of Strains Isolated From Different Wards and Samples & & \\
\hline Clinical Samples & ICU & Respiration Medicine & Burn and Plastic Surgery & Total in the Same Samples \\
\hline Blood culture & 33 & 31 & 27 & 91 \\
Sputum culture & 15 & 18 & - & 33 \\
Wound swab & - & - & 16 & 16 \\
Total in the same department & 48 & 49 & 43 & 140 \\
\hline
\end{tabular}

\begin{tabular}{|c|c|}
\hline House-Keeping Genes Primer & Sequences ( $\left.5^{\prime}-3^{\prime}\right)$ \\
\hline \multicolumn{2}{|l|}{ gltA } \\
\hline $\mathrm{F}$ & AATTTACAGTGGCACATTAGGTCCC \\
\hline $\mathrm{R}$ & GCAGAGATACCAGCAGACATACACG \\
\hline \multicolumn{2}{|l|}{ gyrB } \\
\hline $\mathrm{F}$ & GAATGCTGGTGTACGTATCG \\
\hline $\mathrm{R}$ & ACGCTCAACGTTCAGGATCT \\
\hline \multicolumn{2}{|l|}{ gdhB } \\
\hline $\mathrm{F}$ & GTTAACCGAACGTGCAACTG \\
\hline $\mathrm{R}$ & GCATAGGCATAACCACTGTC \\
\hline \multicolumn{2}{|l|}{ RecA } \\
\hline $\mathrm{F}$ & CCTGAATCTTCCGGTAAAAC \\
\hline $\mathrm{R}$ & GTTTCTGGGCTGCCAAACATTAC \\
\hline \multicolumn{2}{|l|}{ cpn60 } \\
\hline $\mathrm{F}$ & ACTAACGACATCGCAGGTGA \\
\hline $\mathrm{R}$ & CGCGATGTCTTGAAGCATTG \\
\hline \multicolumn{2}{|l|}{ gpi } \\
\hline $\mathrm{F}$ & AATACCGTGGTGCTACGGG \\
\hline $\mathrm{R}$ & ATCGAAATTACCAAACGAAGGTT \\
\hline \multicolumn{2}{|l|}{ rpoD } \\
\hline $\mathrm{F}$ & ATCGAAATTACCAAACGAAGGTT \\
\hline $\mathrm{R}$ & ACGACAGACCCTGTACGTATGTA \\
\hline
\end{tabular}

\footnotetext{
a Abbreviations: $\mathrm{F}$, forward primer; and R, reverse primer.
} 
Qian Yet al.

\subsection{Analysis of Drug Resistance and Related Genes}

The evaluation of antibiotic-resistance was induced by Kirby-Bauer test (K-B) (10). Briefly, the isolates for test were uniformly swabbed across a culture plate. A disk was impregnated with the antibiotic-compounds and then placed on the plate. Concentrations of the compound were the highest next to the disk and decreased as distance from the disk increased. Once the compound was effective against the bacteria at a certain concentration, no colonies would grow at the point where the concentration in the agar was greater than the effective concentration, i. e. the inhibition zone. This could be used to estimate the sensitivity of the bacteria to the antibiotic. Details are shown in Ref 6. Pseudomonas aeruginosa ATCC27853 and Escherichia coli ATCC25922 were used as the quality-control isolates (5).

The clinical and laboratory standards institute (CLSI) standard was selected as the standard evaluation. The drug resistance ability was evaluated by studying the minimum inhibitory concentration (MIC) by agar dilution method. Bacteria were planted in agar plate with different antibiotic concentrations. The MIC results of 140 strains against different antibiotics were studied. The antibiotics used in the study were as follows: Imipenem (IMP), aztreonam (ATM), cefepime (FEP), cefoperazone (CFP), cefotaxime (CTX), piperacillin (PIP), amikacin (AMK), and ciprofloxacin (CIP) (7). All drugs used in the test were bought from Sigma Corporation, US. Resistance inducing genes were identified by PCR. The target genes included methicillinase (oxa-23, oxa-24, oxa-51), carbapenemase (sim, imp, spm), type I integrase intl 1, and sulfonamides-resistance gene (qacE $\Delta 1$-sul1). The sequences of the primers of these genes are listed in Table 3.

\subsection{Statistical Analysis}

Data were analyzed by SPSS software version17.0. Chisquare test was used to compare enumeration data between different groups while Student's t-test for measurement data. One-way analysis of variance was used to analyze dependency of drug resistance and gene expression. P value $<0.05$ was considered as the level of significance.

\begin{tabular}{|c|c|}
\hline Target Genes Primer & Sequence (5'-3') \\
\hline \multicolumn{2}{|l|}{ oxa-23 } \\
\hline $\mathrm{F}$ & GATGTGTCATAGTATTCGTCGT \\
\hline $\mathrm{R}$ & TCACAACAACTAAAAGCACTGT \\
\hline \multicolumn{2}{|l|}{ oxa-24 } \\
\hline $\mathrm{F}$ & ATGAAAAAATTTATACTTCCTATATTCAGC \\
\hline $\mathrm{R}$ & TTAAATGATTCCAAGATTTTCTAGC \\
\hline \multicolumn{2}{|l|}{ oxa-51 } \\
\hline $\mathrm{F}$ & TAATGCTTTGATCGGCCTTG \\
\hline $\mathrm{R}$ & TGGATTGCACTTCATCTTGG \\
\hline \multicolumn{2}{|l|}{$\operatorname{sim}$} \\
\hline $\mathrm{F}$ & TACAAGGGATTCGGCATCG \\
\hline $\mathrm{R}$ & TAATGGCCTGTTCCCATGTG \\
\hline \multicolumn{2}{|l|}{ imp-1 } \\
\hline $\mathrm{F}$ & ATCCAAGCAGCAAGCGCGTTA \\
\hline $\mathrm{R}$ & AGGCGTGCTGCTGCAACGACTTGT \\
\hline \multicolumn{2}{|l|}{ spm } \\
\hline $\mathrm{F}$ & ATTGGTCTATTTGACCGCGTC \\
\hline $\mathrm{R}$ & TGCTACTCAACGACTGAGCG \\
\hline \multicolumn{2}{|l|}{ intl1 } \\
\hline $\mathrm{F}$ & CAGTGGACATAAGCCTGTTC \\
\hline $\mathrm{R}$ & CCCGAGGCATAGACTGTA \\
\hline \multicolumn{2}{|l|}{ qacE $\Delta 1$-sul1 } \\
\hline $\mathrm{F}$ & TAGCGAGGGCTTTACCTAAGC \\
\hline $\mathrm{R}$ & ATTCAGAATGCCGAACACCG \\
\hline
\end{tabular}

\footnotetext{
${ }^{a}$ Abbreviations: $F$, forward primer; $\mathrm{R}$, reverse primer
} 
Qian Yetal.

\section{Results}

\subsection{Detection of the Drug-Resistance Ability of the Isolates}

Results of level of antibiotic-resistance of the isolates are shown in Table 4. All the isolates collected from the hospital under study had a certain level of resistance to all kinds of antibiotics. However, ATM and IMP were still relatively the most effective antibiotics. In order to further evaluate the properties of the isolates, a standard was set to categorize the isolates into three groups (12). Group I, isolates resistant to more than four types of antibiotics; Group II, isolates resistant to three or four types of antibiotics; Group III isolates resistant to less than three types of antibiotics. The categorization results are listed in Table 4. It was identified that among the isolates, the ones isolated from blood had the highest level of drugresistance. Besides, among the three departments, species isolated from the ICU had the highest level of drugresistance. Table 5 present distribution of the isolates in different clinical departments and Clinical Specimens.

\subsection{Results of Multilocus Sequence Typing}

According to MLST analysis, two types of AB strains were sorted and identified from the clinical samples; ST208 and ST218. The detailed distribution of the two strains in clini- cal departments and specimens are listed in Table 6. The ST208 isolates were distributed mainly in ICU and respiration medicine; while ST218 in the burn and plastic surgery department. No significant correlation was found between the types and the sources of the clinical samples ( $P$ value $>0.05)$. But the departments where the strains came from seemed to be significantly related (Pvalue $<0.05$ ). According to the results of K-P drug sensitive test, the strains were divided into two groups, which were carbapenem resistant: the carbapenem resistant $A B(C R A B)$ and the carbapenem sensitive $A B$ (CSAB); $70.21 \%$ of ST208 and $69.21 \%$ of ST218 included CRAB strains, while $15.22 \%$ of ST208 and $84.78 \%$ of ST218 were CSAB. ST208 strains isolated from ICU and respiration medicine department were mainly CRAB; while ST218 strains isolated from burn and plastic surgery departments were mainly CSAB.

\subsection{Detection of Drug-Resistance Genes Expression}

PCR tests revealed that the prevalence of resistance inducing genes were as follows: oxa-23 81.3\%, oxa-51 61.3\%, sim 18.8\%, oxa-24 5.3\%, imp 12.3\%, intl157.9\% and qacE $\Delta 1$-sul1 $61.2 \%$. Further study and compartment were conducted on $\mathrm{CRAB}$ and CSAB. The results are listed in Table 7. Results of the current study showed that ST208/CRAB/oxa-23, 51 is the main molecular phenotype of the CRAB strains in the hospital. Drug-resistance property of the isolates was related to the expression of oxa-23 and 51 (P value $<0.05$ ). Meanwhile,

Table 4. Minimum Inhibitory Concentrations of the AB Isolates ${ }^{a}$

\begin{tabular}{|c|c|c|c|c|}
\hline Antibiotics & MIC $_{\text {range }}$ & $\mathrm{MIC}_{50}$ & $\mathrm{MIC}_{90}$ & Resistance Rate, \% \\
\hline IMP & $0.5-512$ & 32 & 512 & 43.1 \\
\hline ATM & $0.25-256$ & 16 & 256 & 31.8 \\
\hline FEP & $0.03-512$ & 16 & 512 & 62.3 \\
\hline CFP & $0.03-512$ & $>512$ & $>512$ & 100.0 \\
\hline PIP & $0.5-512$ & 512 & $>512$ & 89.4 \\
\hline AMK & $0.5-512$ & $>512$ & $>512$ & 100.0 \\
\hline CIP & $0.03-512$ & $>512$ & $>512$ & 100.0 \\
\hline CTX & $0.03-512$ & 256 & $>512$ & 67.4 \\
\hline
\end{tabular}

a Abbreviations: AMK, amikacin; ATM, aztreonam; CFP, cefoperazone; CIP, ciprofloxacin; CTX, cefotaxime; FEP, cefepime; MIC, minimum inhibitory concentration; PIP, piperacillin; MIC $_{50}$, minimum inhibitory concentration required to inhibit the growth of $50 \%$ of organisms; and MIC $_{90}$, minimum inhibitory concentration required to inhibit the growth of $90 \%$ of organisms.

Table 5. Distribution of the Isolates ${ }^{\mathrm{a}, \mathrm{b}}$

\begin{tabular}{|c|c|c|c|c|c|c|}
\hline \multirow{2}{*}{ Groups c } & \multicolumn{3}{|c|}{ Clinical Departments } & \multicolumn{3}{|c|}{ Clinical Specimens } \\
\hline & ICU & Res & Bur and Pla & Blood & Sputum & Wound \\
\hline I & $39(81.25)$ & $26(53.06)$ & $18(41.86)$ & $58(63.74)$ & $19(57.58)$ & $6(37.50)$ \\
\hline II & $8(16.67)$ & $21(42.86)$ & $22(51.16)$ & $29(31.87)$ & $14(42.42)$ & $8(50.00)$ \\
\hline III & $1(2.08)$ & $2(4.08)$ & $3(6.98)$ & $4(4.40)$ & $0(0.00)$ & $2(12.50)$ \\
\hline Total & $48(100)$ & $49(100)$ & $43(100)$ & $91(100)$ & $33(100)$ & $16(100)$ \\
\hline
\end{tabular}

a Abbreviations: Bur and Pla; department of burn and plastic surgery; ICU, intensive care unit; and Res, department of respiratory medicine.

b Number refers to the number of the isolates from a certain department or samples. Percentage refers to the rate in total number; for example, 39 (81.25) means 39 cases out of $48,39 / 48=81.25 \%$.

C Groups; the groups I, II, III refer to the group classified by the study group. 
Qian Yetal.

Table 6. MLST Results of the Strains ${ }^{a, b}$

\begin{tabular}{|c|c|c|c|c|c|c|}
\hline \multirow[t]{2}{*}{ ST Type } & \multicolumn{3}{|c|}{ Spatial Distributions } & \multicolumn{3}{|c|}{ Tissue Distribution } \\
\hline & ICU & Res & Bur and Pla & Blood & Sputum & Wound Surface \\
\hline ST208 & $40(83.3)^{c}$ & $30(61.22)^{c}$ & $3(69.77)$ & $48(52.75)$ & $24(72.72)$ & $0(0)$ \\
\hline ST218 & $8(16.67)$ & $19(38.78)$ & $40(93.02)^{\mathrm{C}}$ & $43(47.25)$ & $9(27.27)$ & $16(100)$ \\
\hline Total & $48(100)$ & $49(100)$ & $43(100)$ & $91(100)$ & $33(100)$ & $16(100)$ \\
\hline
\end{tabular}

Table 7. Expressions of the Antibiotic-Resistance Inducing Genes in CRAB and CSAB Strains ${ }^{\text {a }}$

\begin{tabular}{lcc}
\hline Types & CRAB & CSAB \\
\hline oxa-23 & $103(90.34)^{\mathrm{b}}$ & $11(9.65)$ \\
oxa-24 & $7(100)^{\mathrm{b}}$ & $0(0)$ \\
\hline oxa-51 & $72(83.72)^{\mathrm{b}}$ & $14(16.28)$ \\
sim & $21(80.77)^{\mathrm{b}}$ & $5(19.23)$ \\
imp & $9(52.94)$ & $8(47.06)$ \\
intl1 & $43(53.08)$ & $38(46.91)$ \\
qacE $\Delta 1-$ sul1 & $40(46.51)$ & $43(53.49)$ \\
\hline
\end{tabular}

a Abbreviations: CRAB, carbapenem-resistant AB; and CSAB, carbapenem-susceptible AB.

$\mathrm{b}$ There are significant differences in this group CRAB compared with those in CSAB, P value $<0.05$.

Table 8. Expression of Drug-Resistance Inducing Genes in ST208 and ST218 Strains ${ }^{\text {a }}$

\begin{tabular}{lcccc}
\hline Strain & \multicolumn{4}{c}{ Genes } \\
\cline { 2 - 5 } & oxa-23 & oxa-51 & intl1 & $\begin{array}{c}\text { qacE 41- } \\
\text { sul1 }\end{array}$ \\
\hline ST208 & $73 / 73^{\mathrm{b}}$ & $70 / 73^{\mathrm{b}}$ & $46 / 73$ & $44 / 73$ \\
ST218 & $41 / 67$ & $16 / 67$ & $35 / 67$ & $39 / 67$ \\
\hline
\end{tabular}

a Data are presented as number of the isolates (which express the gene) per number of the stains

$\mathrm{B}$ There are significant differences in ST208 compared with ST218, P < 0.05 .

in ST208, there were more chances of expressions of oxa-23, 51 compared with those in ST218 (P value < 0.05). Details are shown in Tables 7 and 8.

\section{Discussion}

Acinetobacter baumannii is widely distributed in the environment, human body and surfaces (12). It is the most common pathogenic bacteria which cause nosocomial infections (7). Studies show that growing numbers of carbapenem-resistant pathogenic bacteria, such as CRAB are now appearing. The prevention and treatment of CRAB is an urgent issue to control nosocomial infections (1315). In the current study, all strains of A. baumannii were resistant to antibiotics in different degrees (Table 4). In fact, it is a common phenomenon in hospitals in China
(9). Moreover, it was found that distribution of the CRAB isolates were related to the wards where they came from (Table 5). Isolates from ICU and department of respiratory medicine had higher levels of antibiotic-resistance than the strains from the surgery department. It may be due to the long-term use of antibiotics in the former wards (16-18). This finding showed that the distribution of CRAB may be affected by the therapeutic methods. Besides, authors further studied the types of the isolates in the air and hands of the doctors and nurses in the wards. It was found that in ICU only ST208 existed; while in department of burn and plastic surgery only ST218 was detected. Considering the fact that these two departments were physically separated, it was speculated that the two different isolates may have different origins and they were separated by the spatial distance and were only epidemic in each section.

Currently, techniques for molecular cloning and detection are widely used to study the types of certain isolates $(3,10-13)$. In the current study a new technique, MLST, was used. MLST classified the strains according to the results of accurate detection of the sequences of nucleic acid from the isolates (19). The results were highly repeatable and stable. Two isolates, ST208 and ST218 were found in the hospital, and each one had its own "territory" (Table 6). This finding showed that a certain kind of bacteria would be dominant in a certain section.

The existence of anti-carbapenemase enzymes was the key factor to the prevalence of multidrug-resistant bacteria (20-22). Previous reports showed that 
five types of anti-carbapenemase enzymes, oxa-23, 51, sim, imp and spm could be detected in CRAB (23). The current study found that OXA-23 and 51 were the most prevalent genes in the hospital. Since these antibiotic resistance inducing genes were all expressed in plasmid, their distribution was determined by gene mutation and combined effect of the antibiotics used in a certain place. Thus, the final results of the distribution are the "natural selection" in the certain therapeutic milieu in the hospital. Identification of the types and genes of the isolates in a certain ward would be important to restrict the prevalence. Moreover, although many microbiologists believe that the nuclear-DNA is not related to the plasmid-DNA in bacteria, the current study findings indicated that in ST208 there were more chances for the existence of oxa-23, 51 (Table 8). The fact revealed that there might be certain connections between the nuclear-gene types and the appearance of plasmid genes. The mechanism of this phenomenon is worthy of further studies.

In conclusion, there may be different isolates of $A B$ in different wards since these departments were physically separated. The levels of antibiotic-resistance were also quite different in the isolates from different locations of the human body. Species isolates from blood culture had the highest level of antibiotic-resistance. ST208 and the ST218 were the epidemic isolates of $A B$, their abilities of drug-resistance were due to the existence of oxa-23, oxa51, intl 1 and qace $\Delta 1$-sul 1 . Hospital managers should take measures to supervise the epidemiology and types of the CRAB isolates. The study of the molecular epidemiology of these isolates from different wards and specimens were significantly important. Besides, further studies on the connections between the plasmid gene expression and types of the isolates are required.

\section{Acknowledgements}

Authors are grateful to clinical departments of Shaoxing People's hospital for their help to collect clinical samples.

\section{Author's Contributions}

Qi Liu: original idea, the protocol and abstract development and data analysis; Yaner Qian and Xuejun Dong: did manuscript preparation; Zongxin Wang and Guocan Yang: protocol development and data analysis.

\section{Founding/Support}

This work was supported by the Shaoxing Science and Technology Project, No. 2012B70058, Shaoxing, Zhejiang Province, China.

\section{References}

1. Peleg AY, Seifert H, Paterson DL. Acinetobacter baumannii: emergence of a successful pathogen. Clin Microbiol Rev.2008;21(3):538-82.

2. Haydel SE, Remenih CM, Williams LB. Broad-spectrum in vitro an- tibacterial activities of clay minerals against antibiotic-susceptible and antibiotic-resistant bacterial pathogens. J Antimicrob Chemother. 2008;61(2):353-61.

3. Thong KL, Lai MY, Teh C, Chua KH. Simultaneous detection of methicillin-resistant Staphylococcus aureus, Acinetobacter baumannii, Escherichia coli, Klebsiella pneumoniae and Pseudomonas aeruginosa by multiplex PCR. Trop Biomed 2011;28(1):21-31.

4. Mardani M. Pan-resistant Acinetobacter baumannii: Is there any available alternative therapy? Arch Clin Infect Dis. 2011;6(2):65.

5. Guo-xin M, Dan-yang S, Xi-zhou G, Jun-chang C, Rui W, Zhi-gang C, et al. Laboratory to Clinical Investigation of Carbapenem Resistant Acinetobacter baumannii Outbreak in a General Hospital. Jundishapur J Microbiol. 2014;7(1):e13120.

6. Higgins PG, Dammhayn C, Hackel M, Seifert H. Global spread of carbapenem-resistant Acinetobacter baumannii. I Antimicrob Chemother. 2010;65(2):233-8.

7. Japoni-Nejad A, Sofian M, van Belkum A, Ghaznavi-Rad E. Nosocomial outbreak of extensively and pan drug-resistant Acinetobacter baumannii in tertiary hospital in central part of Iran Jundishapur J Microbiol. 2013;6(8):e9892.

8. Mardani M. Approaches to Treat Patients Infected With MultiDrug-Resistant Acinetobacter baumannii. Arch Clin Infect Dis. 2014;8(1):1-2

9. Dantas G, Sommer MO, Oluwasegun RD, Church GM. Bacteria subsisting on antibiotics. Science. 2008;320(5872):100-3.

10. Gautam V, Singhal L, Arora S, Jha C, Ray P. Reliability of Kirby-Bauer disk diffusion method for detecting carbapenem resistance in Acinetobacter baumannii-calcoaceticus complex isolates. Antimicrob Agents Chemother. 2013;57(4):2003-4.

11. Woo PC, Tsang AK, Wong AY, Chen H, Chu J, Lau SK, et al. Analysis of multilocus sequence typing schemes for 35 different bacteria revealed that gene loci of 10 bacteria could be replaced to improve cost-effectiveness. Diagn Microbiol Infect Dis. 2011;70(3):316-23.

12. Wang F, Zhu D, Hu F, et al. Surveillance of bacterial resistance in China. Chin J of Infect Chemother . 2008;5(3):2009-5.

13. Ecker JA, Massire C, Hall TA, Ranken R, Pennella TT, Agasino Ivy $\mathrm{C}$, et al. Identification of Acinetobacter species and genotyping of Acinetobacter baumannii by multilocus PCR and mass spectrometry. J Clin Microbiol. 2006;44(8):2921-32.

14. Dijkshoorn L, Nemec A, Seifert H. An increasing threat in hospitals: multidrug-resistant Acinetobacter baumannii. Nat Rev Microbiol. 2007;5(12):939-51.

15. Fu Y, Zhou J, Zhou H, Yang Q, Wei Z, Yu Y, et al. Wide dissemination of OXA-23-producing carbapenem-resistant Acinetobacter baumannii clonal complex 22 in multiple cities of China. J Antimicrob Chemother. 2010;65(4):644-50.

16. Martinez JL. Antibiotics and antibiotic resistance genes in natural environments. Science. 2008;321(5887):365-7.

17. Killgore G, Thompson A, Johnson S, Brazier J, Kuijper E, Pepin J, et al. Comparison of seven techniques for typing international epidemic strains of Clostridium difficile: restriction endonuclease analysis, pulsed-field gel electrophoresis, PCR-ribotyping, multilocus sequence typing, multilocus variable-number tandemrepeat analysis, amplified fragment length polymorphism, and surface layer protein A gene sequence typing. J Clin Microbiol. 2008;46(2):431-7.

18. Mardani M. Pan-resistant Acinetobacter baumannii: Is there any available alternative therapy? Arch Clin Infect Dis 2011;6(2):65.

19. Ragimbeau C, Schneider F, Losch S, Even J, Mossong J. Multilocus sequence typing, pulsed-field gel electrophoresis, and fla short variable region typing of clonal complexes of Campylobacter jejuni strains of human, bovine, and poultry origins in Luxembourg. Appl Environ Microbiol. 2008;74(24):7715-22.

20. Mardani M. Approaches to Treat Patients Infected With MultiDrug-Resistant Acinetobacter baumannii. Arch Clin Infect Dis. 2014;8(1):1-2.

21. Chouchani ET, Hurd TR, Nadtochiy SM, Brookes PS, Fearnley IM, Lilley KS, et al. Identification of S-nitrosated mitochondrial proteins by S-nitrosothiol difference in gel electrophoresis (SNO-DIGE): implications for the regulation of mitochondrial function by reversible S-nitrosation. Biochem J. 
Qian Yet al.

2010;430(1):49-59.

22. Walsh TR. Emerging carbapenemases: a global perspective. Int $J$ Antimicrob Agents. 2010;36 Suppl 3:S8-14.

23. Karmostaji A, Najar Peerayeh SH, Salmanian AH. Distribution of
OXA-Type Class D $\beta$-Lactamase Genes Among Nosocomial Multi Drug Resistant Acinetobacterbaumannii Isolated in Tehran Hospitals.JundishapurJ Microbiol.2013;6(5): e8219. 